\title{
Um caso de demência multifatorial
}

Maria João Albuquerque da Silva Viveiros, ${ }^{1}$ Andreia Felizardo, ${ }^{2}$ Mariana Farinha Alves ${ }^{3}$

\section{RESUMO}

Introdução: A demência pode apresentar-se com deterioração de um ou vários domínios cognitivos, caracterizando-se pelo impacto e desadaptação nas atividades de vida diária (AVD). A apresentação deste caso clínico justifica-se pela incidência crescente deste diagnóstico e pela importância do reconhecimento atempado de fatores causais reversíveis para o mesmo, que permita a realização de tratamento dirigido e a minimização de complicações.

Descrição do Caso: Homem de 70 anos, solteiro, ex-fumador, com antecedentes de alcoolismo e de comportamentos sexuais de risco. É trazido pela filha à consulta por um quadro com quatro anos de evolução de alteração comportamental, com episódios de heteroagressividade, discurso incoerente, perda progressiva de autonomia e autonegligência e descoordenação motora associada a alterações da marcha com quedas frequentes. Destaca-se a deteção, duas semanas antes da data da consulta, de lesão cutânea escrotal com $4 \times 2 \mathrm{~cm}$ de diâmetro e $2 \mathrm{~cm}$ de espessura, de consistência elástica, indolor, ulcerada, compatível com goma sifilítica. Por suspeita de sífilis terciária é encaminhado para o serviço de urgência (SU), com posterior internamento e confirmação de neurossífilis, tendo cumprido terapêutica prolongada com penicilina endovenosa (EV), com melhoria do quadro neurológico. Três meses depois é reinternado por reativação de neurossífilis e medicado com penicilina, seguindo-se, após alta, um agravamento do seu estado clínico, com caquexia, diminuição global da força muscular mais evidente ao nível dos membros inferiores, disartria marcada e disfagia progressiva. Faleceu 11 meses depois, por pneumonia associada aos cuidados de saúde.

Comentário: Neste caso clínico destaca-se a presença de uma causa potencialmente reversível de demência: neurossífilis. Após tratamento verificou-se uma melhoria parcial do quadro; contudo, sem completa recuperação e com posterior deterioração progressiva. Este aspeto justifica-se pelo diagnóstico tardio e pela presença concomitante de outros fatores etiológicos para a síndroma demencial. Pelo conhecimento dos antecedentes pessoais, do contexto sociofamiliar e do estado prévio do seu doente, o médico de família encontra-se numa posição privilegiada para o diagnóstico precoce e orientação destas situações.

Palavras-chave: Demência; Declínio cognitivo; Neurossífilis; Cuidados de saúde primários.

\section{INTRODUÇÃO}

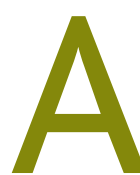

demência é uma síndroma clínica caracterizada por uma perturbação da função cognitiva. Pode apresentar-se com uma deterioração de um único ou de vários domínios. Os sinais e sintomas vão desde a dificuldade de memorização, distúrbios de linguagem e alterações psicológicas ou psiquiátricas, que se caracterizam pelo impacto e desadaptação nas AVD, desde básicas a instrumentais, podendo comprometer também a autonomia do utente. A demência, embora de etiologia frequentemente mul-

1. Médica Interna de Medicina Geral e Familiar. USF Santo António da Charneca, ACeS Arco Ribeirinho. Barreiro, Portugal.

2. Médica Interna de Medicina Geral e Familiar. USF Eça, ACeS Arco Ribeirinho. Barreiro, Portugal.

3. Médica Interna de Medicina Geral e Familiar. USF Santo António da Charneca, ACeS Arco Ribeirinho. Barreiro, Portugal. tifatorial, pode dividir-se em dois subgrupos segundo a sua causa: primária degenerativa, com perda de massa neuronal, e secundária, onde existe outra patologia de base que se manifesta por demência, desencadeada por dano neuronal. Sabe-se que, em 2015, cerca de $47 \mathrm{mi}-$ lhões de pessoas no mundo viviam com demência, estimando-se que este valor triplique em 2050. ${ }^{1}$ Além disso, Portugal é o quarto país da OCDE com mais casos de demência, onde se prevê que mais de uma em cada 25 pessoas venha a ter este diagnóstico em $2050 .{ }^{2}$ Assim, as autoras consideram pertinente a divulgação deste caso clínico. O conhecimento dos fatores precipitantes e, principalmente, dos reversíveis tem impacto no diagnóstico precoce e, consequentemente, repercussões sobre a eficácia do tratamento dirigido, minimizando as complicações e preservando a qualidade de vida. 


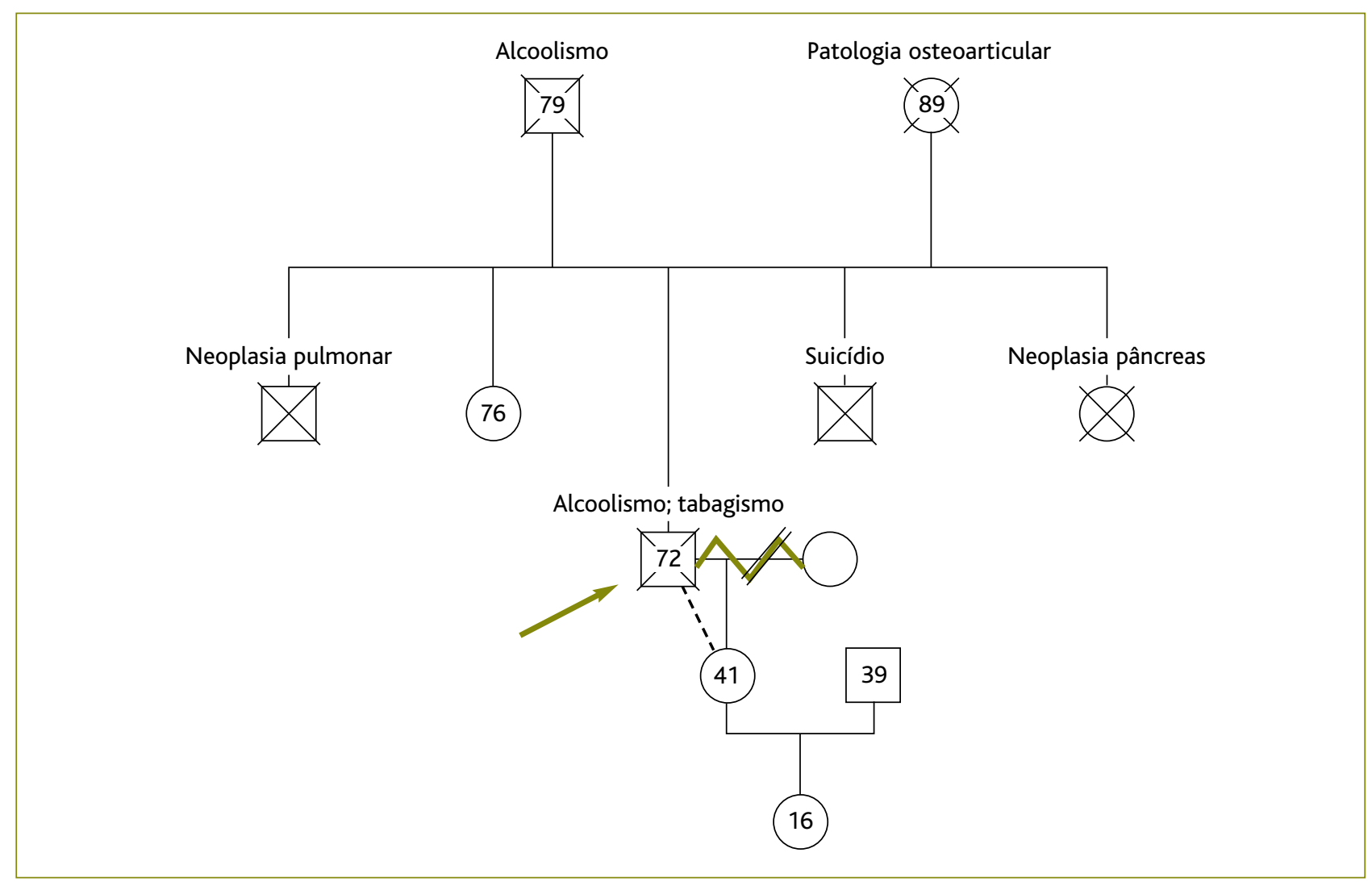

Figura 1. Genograma.

\section{DESCRIÇÃO DO CASO}

Identificação e história pessoal

Descreve-se o caso de um homem de 70 anos de idade, solteiro e caucasiano. Tem o $4 .^{\circ}$ ano de escolaridade e está reformado da profissão de serralheiro desde os 62 anos de idade.

Trata-se de um doente ex-fumador desde há três meses, com uma carga tabágica de 55 unidades maço-ano (UMA) e com antecedentes de alcoolismo, caracterizados pelo consumo médio diário de 15 unidades de bebida padrão desde os 32 anos de idade, abstinente desde há três anos. Tem história conhecida de comportamentos sexuais de risco, com relações sexuais desprotegidas ao longo da sua vida, com múltiplas parceiras. Sem acompanhamento regular pelo médico de família, sem história de viagens recentes. O seu plano nacional de vacinação está atualizado. Não possui medicação crónica.

\section{Caracterização familiar}

O doente é o terceiro de uma fratria de cinco irmãos. É solteiro, cumpriu o primeiro ciclo de escolaridade básica, tem uma filha de 41 anos de idade, casada, com antecedentes conhecidos de asma, e uma neta de 16 anos de idade (Figura 1). Reside sozinho, constituindo uma família unitária, de classe socioeconómica média-baixa segundo a escala de Graffar. A história é fornecida pela filha que retrata, no passado, uma situação familiar instável, com vários momentos disruptivos entre ambos os progenitores, com episódios de violência doméstica, que culminaram no abandono, por parte da mãe, quando esta tinha quatro anos de idade, tendo ficado aos cuidados do pai e avó paterna. A filha recorda vários contactos fugazes entre o doente e diferentes parceiras ao longo de toda a sua infância, tendo-se distanciado após atingir a idade adulta. 


\section{História da doença atual}

\section{Consulta programada (09/10/2018)}

O doente é trazido à consulta na unidade de saúde familiar (USF) pela filha, por um quadro insidioso com quatro anos de evolução. O quadro caracterizava-se inicialmente por alterações do comportamento, com heteroagressividade caracterizada por episódios de irritabilidade e agressividade verbal dirigida aos familiares. Nestes episódios apresentava também discurso incoerente e descoordenação motora com dismetria, marcha de base alargada e arrastada. Não aparentava alterações da perceção, nem crítica para as alterações comportamentais. Não se apuraram alterações da memória mantidas. Este quadro clínico foi-se agravando, com perda progressiva na capacidade de execução das AVD básicas, como: dificuldade em alimentar-se, descuido pela higiene pessoal e quedas frequentes no domicílio por alterações da marcha e dependência total para as atividades instrumentais da vida diária. Apesar da insistência por parte da filha em procurar cuidados médicos, o doente mantinha recusa em procurar apoio, reagindo agressivamente a esta sugestão. Quinze dias antes da data da consulta na USF, o doente tinha sido levado ao SU pela filha, após queda no domicílio com traumatismo craniano, tendo sido institucionalizado num lar de idosos após alta hospitalar. À data da consulta na USF, a filha manifesta preocupação pela presença de uma lesão cutânea nos genitais externos, detetada durante os cuidados de higiene no lar aquando do seu ingresso no mesmo.

Ao exame objetivo, o doente apresenta-se emagrecido, com idade aparente superior à real, mucosas ligeiramente descoradas e desidratadas. Ao exame neurológico apresenta-se vígil, pouco colaborante e desorientado no tempo, com discurso lentificado, incoerente e pouco percetível. Destaca-se ainda ataxia da marcha e tremor das extremidades, sem outras alterações. O Mini-Mental State Examination (MMSE) não foi realizado por falta de colaboração do doente. Na região escrotal apresenta uma lesão com cerca de $4 \times 2 \mathrm{~cm}$ de diâmetro e $2 \mathrm{~cm}$ de espessura, de consistência elástica, indolor, ulcerada, revestida com tecido de granulação, compatível com goma sifilítica (Figuras 2 e 3). Por suspeita de sífilis terciária, o doente é encaminhado para o SU.

No SU, a 09/10, realiza avaliação analítica, com serologias virais (VIH, vírus da hepatite $\mathrm{C}$ e teste não tre- ponémico), das quais se destaca o teste Venereal $D i$ sease Research Laboratory (VDRL) positivo a 1:64 diluições (dils.). Efetua ainda tomografia computorizada crânio-encefálica (TC-CE), que não objetiva lesões vasculares recentes ou lesões ocupando espaço. Assim, é internado no serviço de medicina interna com suspeita de neurossífilis, para estudo dirigido e tratamento.

\section{Internamento (10/10/2018 - 28/1 1/2018)}

Durante o internamento realiza punção lombar para colheita de líquido cefalorraquidiano (LCR) por suspeita de neurossífilis, com pesquisa de antigénios, exame citoquímico, microbiológico e serologias. Da análise do LCR destaca-se: proteinorráquia de $69,5 \mathrm{mg} / \mathrm{dl}$ e VDRL no LCR positivo a 1:2 dils. Não foi possível pesquisar antigénios por amostra insuficiente (Tabela 1a). Após confirmação de neurossífilis inicia tratamento EV com penicilina G $24 \mathrm{MUI} /$ dia. Durante o internamento mantém-se hemodinamicamente estável, sem alterações de novo ao exame objetivo, com melhoria da lesão genital. Realiza ressonância magnética crânio-encefálica, que mostra alterações de natureza microangiopática incipientes, bem como padrão de discreta atrofia cerebral cortical difusa. A resposta à terapêutica foi monitorizada pelo teste não treponémico Rapid Plasma Reagin (RPR), que se manteve consistentemente reativo a 1:64 dils. Assim, manteve terapêutica com penicilina durante 37 dias, tendo-se verificado a resolução do quadro infecioso ao $40 .^{\circ}$ dia de internamento, após RPR não reativo.

\section{Consultas de seguimento}

Em consulta de seguimento na USF, três semanas após alta hospitalar, apresenta-se orientado no tempo e no espaço, com discurso pobre, mas percetível, mais coerente e menos lentificado, embora sem recuperação completa da marcha e da independência para as AVD, mantendo-se em acompanhamento em consultas de medicina interna e de neurologia.

$\mathrm{Na}$ consulta de controlo de medicina interna, a 08/02/2019, apresenta RPR novamente reativo para 64 dils., tendo sido agendada nova punção lombar, que se realiza a 18/02/2019. Desta análise (Tabela $1 b$ ) destaca-se a presença de proteinorráquia $>200 \mathrm{mg} / \mathrm{dL}$ (não confirmada por LCR em quantidade insuficiente) e VDRL novamente positivo a 2 dils. 


\begin{tabular}{|c|c|c|}
\hline Exames laboratoriais & a) & b) \\
\hline Hemoglobina $(\mathrm{Hb})$ & $12,8 \mathrm{~g} / \mathrm{dl}$ & não aplicável \\
\hline Hematócrito (Htc) & $38,50 \%$ & não aplicável \\
\hline Volume globular médio (VGM) & $94 \mathrm{fL}$ & não aplicável \\
\hline Leucócitos & $5,90 \times 109$ & não aplicável \\
\hline Ureia & $28 \mathrm{mg} / \mathrm{dL}$ & não aplicável \\
\hline Creatinina & $0,66 \mathrm{mg} / \mathrm{dL}$ & não aplicável \\
\hline AST & $26 \mathrm{UI} / \mathrm{L}$ & não aplicável \\
\hline ALT & $27 \mathrm{UI} / \mathrm{L}$ & não aplicável \\
\hline Fosfatase alcalina (FA) & $80 \mathrm{UI} / \mathrm{L}$ & não aplicável \\
\hline Desidrogenase láctica (LDH) & $197 \mathrm{UI} / \mathrm{L}$ & não aplicável \\
\hline TSH & $0,57 \mathrm{uUI} / \mathrm{mL}$ & não aplicável \\
\hline T3 livre & $2,4 \mathrm{pg} / \mathrm{mL}$ & não aplicável \\
\hline T4 livre & $0,99 \mathrm{ng} / \mathrm{dL}$ & não aplicável \\
\hline VIH I e II & negativo & não aplicável \\
\hline Anticorpo Hbs & $<2,5 \mathrm{UI} / \mathrm{L}$ & não aplicável \\
\hline Vírus hepatite $C$ & negativo & não aplicável \\
\hline VDRL sérico & reativo 64 dils. & não realizado \\
\hline RPR & não realizado & reativo 64 dils. \\
\hline PCR & $4,4 \mathrm{mg} / \mathrm{L}$ & não aplicável \\
\hline LCR (exame citoquímico) & $\begin{array}{l}\text { límpido, } 1 \text { elemento mononucleado; } \\
\text { glicose } 52 \mathrm{mg} / \mathrm{dL} \text {; proteínas 69,5mg/dL; } \\
\text { LDH } 28 \mathrm{UI} / \mathrm{L}\end{array}$ & $\begin{array}{l}\text { ligeiramente hemático, } 4 \text { elementos } \\
\text { celulares } / \mathrm{mm}^{3} \text {, muitos eritrócitos; } \\
\text { glicose } 55 \mathrm{mg} / \mathrm{dL} \text {; proteínas > 200mg/dL; } \\
\text { LDH } 153 \mathrm{UI} / \mathrm{L}\end{array}$ \\
\hline LCR (exame microbiológico) & negativo & negativo \\
\hline LCR (pesquisa de antigénios) & amostra insuficiente & não realizado \\
\hline VDRL LCR & reativo 2 dils. & reativo 2 dils. \\
\hline
\end{tabular}

Legenda: a) primeiro internamento; b) segundo internamento.

\section{Reinternamento (20/02/2019 - 14/03/2019)}

Neste contexto é internado e transferido para o serviço de neurologia, onde inicia novamente terapêutica EV com penicilina, assumindo-se reativação de neurossífilis. Durante o internamento mantém-se orientado na pessoa, desorientado no tempo e espaço, colaborante, com alguns períodos de confusão, marcha de base larga, possível a curtas distâncias, com apoio. Realiza nova TC-CE, sobreponível à anterior. Mantém estado neurológico sobreponível durante o internamen- to, tendo tido alta após cumprir 14 dias de antibioterapia.

\section{Evolução}

Após alta observa-se o agravamento gradual do seu estado clínico, caracterizado por caquexia, disartria marcada com evolução para discurso impercetível, disfagia progressiva, diminuição da força muscular, embora sem fasciculações, ortostatismo possível com apoio bilateral com evolução para incapacidade para a 
marcha autónoma. No dia 15/02/2020 dá entrada no SU com quadro de dificuldade respiratória grave, com posterior confirmação do seu óbito por insuficiência respiratória devido a pneumonia associada aos cuidados de saúde.

\section{COMENTÁRIO FINAL}

O esclarecimento etiológico de uma síndroma demencial pode constituir um desafio. A maioria tem causa neurodegenerativa, condicionando um compromisso funcional e dependência progressivos. Importa, numa abordagem inicial de um quadro de declínio cognitivo ou perante rápido agravamento do estado prévio, excluir causas potencialmente reversíveis: doenças infeciosas, metabólicas, défices nutricionais, etilismo ou anomalias estruturais. ${ }^{3}$ Perante este diagnóstico existem características que sugerem a presença de uma possível etiologia reversível, como se verifica no caso apresentado: história de comportamentos de risco; achados no exame neurológico não explicados pelo quadro demencial, neste caso, ataxia da marcha e tremor distal; idade de instalação precoce; flutuação sintomática evidente numa fase inicial do caso apresentado; desempenho nos testes neurocognitivos discordante da história clínica, ${ }^{4}$ (não enquadrável neste caso) e rápido declínio funcional (não enquadrável neste caso).

Neste caso, a neurossífilis foi o principal fator etiológico para a síndroma demencial detetada. Na sua forma tardia, a neurossífilis constitui uma forma de sífilis terciária, rara nos países desenvolvidos desde a generalização do uso de antibióticos. O curso da terapêutica é necessariamente prolongado, tanto pela baixa taxa de replicação do Treponema pallidum como pelo sequestro em regiões do sistema nervoso central (SNC), de difícil penetração dos fármacos. ${ }^{5}$ Em relação ao caso em discussão, após cumprimento de antibioterapia prolongada em regime de internamento, registou-se melhoria global do quadro neurológico. Contudo, não foi constatada a completa recuperação do estado cognitivo nem da independência nas AVD. Este aspeto justifica-se pelo diagnóstico tardio e pela presença concomitante de outros fatores etiológicos para demência, cujas lesões provocadas no SNC são irreversíveis, designadamente: consumo abusivo de bebidas alcoólicas e eventual patologia cerebrovascular. Para além destes, contribuem para o desfecho desfavorável a idade, o estado de desnutrição, o isolamento social e empobrecimento das relações e atividades, com escassa reserva cognitiva prévia.

O presente caso é ilustrativo da potencial natureza multifatorial da síndroma demencial. Face ao progressivo envelhecimento da população portuguesa prevê-se um aumento da prevalência desta patologia. Importa ao médico de família saber abordar estas situações e saber como e quando despistar causas potencialmente reversíveis, nunca minimizando a importância da prevenção e da redução de fatores de risco para a mesma. Pelo conhecimento dos antecedentes pessoais, do contexto sociofamiliar e do estado prévio do seu doente, o médico de família encontra-se numa posição privilegiada para o diagnóstico e orientação destas situações.

\section{REFERÊNCIAS BIBLIOGRÁFICAS}

1. Baumgart M, Snyder HM, Carrillo MC, Fazio S, Kim H, Johns H. Summary of the evidence on modifiable risk factors for cognitive decline and dementia: a population-based perspective. Alzheimers Dement. 2015;11(6):718-26.

2. Organisation for Economic Cooperation and Development. Health at a glance 2019: OECD indicators [homepage]. Paris: OECD Publishing; 2020. Available from: https://doi.org/10.1787/4dd50c09-en

3. Knopman DS, DeKosky ST, Cummings JL, Chui H, Corey-Bloom J, Relkin $\mathrm{N}$, et al. Practice parameter: diagnosis of dementia (an evidence-based review): report of the Quality Standards Subcommittee of the American Academy of Neurology. Neurology. 2001;56(9):1143-53.

4. Day GS. Reversible dementias. Continuum. 2019;25(1):234-53.

5. Workowski KA, Bolan GA. Sexually transmitted diseases treatment guidelines, 2015. MMWR Recomm Rep. 2015;64(RR-03):1-137.

\section{CONFLITO DE INTERESSES}

Os autores declaram não possuir quaisquer conflitos de interesse.

\section{ENDEREÇO PARA CORRESPONDÊNCIA}

Maria João Albuquerque da Silva Viveiros

E-mail: mariaviveiros1990@hotmail.com

https://orcid.org/0000-0002-4097-1587

Recebido em 04-11-2020

Aceite para publicação em 27-05-2021 


\section{ABSTRACT}

\section{A CASE OF MULTIFACTORIAL DEMENTIA}

Introduction: Dementia may present with deterioration of one or several cognitive domains, characterized by impact and maladaptation in the activities of daily living. The presentation of this clinical case is justified by the growing incidence of this diagnosis and the importance of the timely recognition of reversible causal factors for it, allowing for a targeted treatment and the minimization of complications.

Case Description: Single, 70-year-old man, former smoker, with a history of alcoholism and sexual risk behavior. He was brought to the consultation by his daughter because of a four-year history of behavioral change, with episodes of hetero-aggressivity, incoherent speech, self-neglect, progressive loss of autonomy, and motor incoordination associated with gait alterations with frequent falls. The recent detection, two weeks before the consultation date of a scrotal skin lesion, $4 \times 2 \mathrm{~cm}$ in diameter and $2 \mathrm{~cm}$ thick, of elastic consistency, painless, ulcerated, compatible with syphilitic gum, stands out. On suspicion of tertiary syphilis, he was referred to the emergency service, with subsequent admission and confirmation of neurosyphilis, having followed prolonged therapy with intravenous penicillin, with an improvement of the neurological condition. Three months later, he was re-hospitalized for reactivation neurosyphilis and medicated with penicillin, followed, after discharge, by a worsening of his clinical condition, with cachexia, a global decrease of muscle strength more evident in the lower limbs, marked dysarthria, and progressive dysphagia. He died 11 months later from healthcare-associated pneumonia.

Comment: In this clinical case, the presence of a potentially reversible cause of dementia stands out: neurosyphilis. After treatment, there was a partial improvement of the clinical condition, however without complete recovery and with subsequent progressive deterioration. This aspect is justified by the late diagnosis and by the concomitant presence of other etiological factors for dementia syndrome. By knowing the personal antecedents, the socio-familiar context, and the patient's previous condition, the family doctor is in a privileged position for the early diagnosis and orientation of these situations.

Keywords: Dementia; Cognitive decline; Neurosyphilis; Primary health care. 